\title{
Estudio Comparativo entre la Técnica de Anestesia Local Controlada por Computador y la Técnica de Anestesia Local Convencional
}

\author{
Comparative Study Between the Conventional Local Anesthetic Technique \\ and the Computer-controlled Local Anesthetic
}

Barros, T. P.*; Campolongo, G.*; Sevilha, F.*; Duarte, D.*; Borelli Neto, L.* \& Alves, N.**

BARROS, T. P.; CAMPOLONGO, G.; SEVILHA, F.; DUARTE, D.; BORELLI NETO, L. \& ALVES, N. Estudio comparativo entre la técnica de anestesia local controlada por computador y la técnica de anestesia local convencional. Int. J. Odontostomat., 7(2):175-178, 2013.

RESUMEN: El control del dolor es uno de los aspectos más importantes para propiciar al paciente un comportamiento positivo en la consulta odontológica. Sin embargo, esto aún constituye un desafío para el cirujano dentista cuando trata pacientes ansiosos, ya que la administración de la anestesia local es lo que determina que muchos pacientes eviten el tratamiento odontológico. Con el propósito de disminuir la sensación dolorosa en pacientes y promover mayor comodidad, los profesionales han desarrollado un método de anestesia computarizada. El objetivo de esta investigación fue comparar la técnica de anestesia local convencional con la técnica de anestesia local controlada por computador. Participaron 30 pacientes voluntarios adultos que tenían indicación para exodoncia de terceros molares inferiores de ambos lados. Los pacientes, elegidos de forma aleatoria, fueron sometidos a las dos técnicas anestésicas mencionadas (convencional y controlada por computador). Aunque el grado de satisfacción de los profesionales con la técnica anestésica controlada por computador fue significativa, la mayoría declaró preferir el método convencional. No hubo cambios hemodinámicos significativos en cualquiera de los dos métodos utilizados en este estudio. La técnica anestésica controlada por computador se mostró sencilla, eficaz y segura. Los pacientes que participaron en este estudio respondieron mejor al método de anestesia controlada por computador, reportando menos dolor.

PALABRAS CLAVE: anestesia local, anestesia computarizada, control del dolor.

\section{INTRODUCCIÓN}

El control del dolor es uno de los aspectos más importantes para propiciar al paciente un comportamiento positivo en la consulta odontológica (Walter et al., 1996; Wilson, 1996; Guedes Pinto, 2010). Sin embargo, esto aún constituye un desafío para el cirujano dentista cuando trata pacientes ansiosos, ya que la administración de la anestesia local es lo que determina que muchos pacientes eviten el tratamiento odontológico (Johnson \& Primosch, 2003). La anestesia local es un procedimiento a través del cual, por deposición de un agente químico adecuado cerca de los nervios, es posible bloquear la conducción de los impulsos nerviosos. Es eficaz para casi todos los procedimientos dentales, pero puede causar alguna moles- tia durante la inyección, causando temor y ansiedad en muchos pacientes (Alves \& Cândido, 2008; 2012). Primosch \& Brooks (2002) relataron que la inyección rápida de anestésico líquido podría causar distensión del tejido y dañarlo, lo que resultaría en aumento de la sensación dolorosa durante la inyección.

El control del flujo de la inyección en la anestesia local es difícil debido al funcionamiento del sistema tradicional de la jeringa. La jeringa tradicional aplica el anestésico a una tasa influenciada por el control muscular del operador, por la técnica y también por la resistencia del tejido en el sitio de la inyección. Con la jeringa tradicional no es posible controlar la presión y 
el parámetro de volumen de manera precisa. Es técnicamente difícil mantener una tasa de flujo constante cuando se encuentra resistencia en diferentes tejidos utilizando administración manual (Primosch \& Brooks).

El control de la presión y del volumen de la solución anestésica utilizada durante la anestesia local son factores importantes para el éxito de la técnica anestésica, así como la selección de una aguja estrecha y una velocidad de administración del flujo lenta para reducir la presión de distensión del tejido sentida por el paciente (Johnson \& Primosch). El sistema de anestesia local controlado por computador regula estos factores, ya que libera el líquido anestésico con presión y volumen constantes, reduciendo el malestar y el dolor (Primosch \& Brooks). La inyección lenta del anestésico puede ser controlada con mayor precisión con este sistema que con el método tradicional, incluso en las regiones del ligamento periodontal y paladar, donde hay gran resistencia tisular (Johnson \& Primosch). Esto es posible porque las velocidades son preestablecidas (lenta y rápida) y se realizan automáticamente, independiente de la densidad del tejido. La inyección se inicia y se detiene a través de un pedal de control, con eso la atención del operador puede ser totalmente centrada en el posicionamiento y control de la aguja (Primosch \& Brooks).

Estudios clínicos han tratado de investigar la eficacia de la anestesia local controlada por computador para reducir el dolor en la inyección en niños (Gibson et al., 2000; Allen et al., 2002) y los resultados han demostrado que el sistema computarizado reduce significativamente los trastornos del comportamiento relacionados con la anestesia.

El objetivo de la presente investigación fue comparar la técnica convencional con la técnica de anestesia local controlada por computador en pacientes adultos.

\section{MATERIAL Y MÉTODO}

Participaron en este estudio 30 pacientes adultos, voluntarios, asistidos en la disciplina de Cirugía y Traumatología Bucomaxilofacial de la Facultad de Odontología de la Universidad Bandeirante de São Paulo. Todos los pacientes tenían indicación para exodoncia de terceros molares inferiores de ambos lados.
Los pacientes, elegidos de forma aleatoria, fueron sometidos a las dos técnicas anestésicas mencionadas: convencional y la que utiliza inyector anestésico controlado por computador. Se realizó en cada paciente una técnica distinta para cada lado, utilizándose la solución anestésica: Articaína 4\% con adrenalina 1:200.000 (Articaíne - DFL), envasada en cartuchos de vidrio de $1,8 \mathrm{ml}$. Se preparó una tabla de proporcionalidad de la dosis en la cual se utilizó una cantidad estándar de acuerdo con el peso de cada paciente.

El aparato utilizado para la técnica que utiliza inyector anestésico controlado por computador fue el Morpheus ${ }^{\circledR}$ que cuenta con 10 velocidades preprogramadas con variación desde 0,1 hasta $1,0 \mathrm{ml} /$ min. Consiste de una unidad móvil con pantalla interactiva, emite mensajes sonoras, y tiene una punta aplicadora para jeringuilla con diseño que facilita la aplicación. Permite la utilización de cartuchos anestésicos y agujas convencionales.

\section{RESULTADOS}

Participaron de este estudio 30 pacientes. Después de la anestesia fueron interrogados respecto la sensibilidad dolorosa, indicando si habían sentido "mucho dolor", " dolor leve" o "ningún dolor" en cada uno de los métodos de anestesia.

Al principio, fueron evaluados con la técnica convencional usando la jeringa de tipo carpule, donde ningún paciente refirió mucho dolor, $60 \%$ reportaron dolor leve y sólo un $40 \%$ reportaron ningún dolor en el procedimiento. Los mismos pacientes fueron evaluados en el lado contrario, sin embargo utilizando el sistema de anestesia controlado por computador y se les hicieron las mismas preguntas. Ninguno de estos pacientes relató sentir mucho dolor durante la anestesia, el $30 \%$ reportaron sentir dolor leve y el $70 \%$ de estos pacientes reportaron ningún dolor durante el procedimiento.

En relación a cambios hemodinámicos no hubo diferencias importantes entre las dos técnicas. Sólo un $20 \%$ de los pacientes presentaron alteraciones antes del procedimiento en la técnica convencional y un $16,70 \%$ de los pacientes en la técnica controlada por computador. En el postoperatório $13,4 \%$ de los pacientes permanecieron con ligero cambio en la presión arterial, en ambas técnicas, pero no hubo complicaciones. 
Otro aspecto analizado fue el grado de satisfacción del profesional durante la anestesia. Los profesionales no relataron dificultad con cualquiera de las dos técnicas, pero el $40 \%$ demostró una facilidad con la técnica anestésica controlada por computador mientras que los otros $60 \%$ aún prefieren la técnica convencional.

\section{DISCUSIÓN}

La administración de anestesia local, el procedimiento que permite bloquear la conducción de los impulsos nerviosos, es el acto profesional más frecuente en la práctica dental. A pesar de los avances tecnológicos y mejoras significativas en la Odontología, el dolor a la inyección persiste como una fuente de temor y ansiedad para muchos pacientes.

El dolor durante la administración del líquido anestésico puede ser explicado por varios factores, incluyendo la edad, experiencias previas desagradables, estado emocional, ansiedad, entre otros. Una explicación reportada por muchos autores es que la rápida inyección del líquido anestésico podría causar distensión del tejido y dañarlo, lo que resultaría en aumento de la sensación de dolor durante la inyección (Bennett, 1989; Vieira et al., 2000; Primosch \& Brooks). Concordamos con estos autores y recordamos que a veces es difícil el control manual de flujo anestésico, principalmente en los tejidos más rígidos como en la región palatal, en consecuencia se administra más anestésico que el tejido puede absorber, determinando aumento de la presión interna y sensibilidad dolorosa.

Con el propósito de controlar la inyección de solución anestésica y proporcionar una mayor comodidad a los pacientes, surgió en las últimas décadas una técnica de anestesia que tiene la misma función que el método convencional, pero permite la programación antes de la anestesia. Se trata de la técnica de anestesia local controlada por computador, que permite definir la cantidad de anestésico inyectado, el tiempo y el volumen por minuto, dependiendo de la región a ser anestesiada y si el paciente es adulto o niño. Se puede utilizar en cualquier técnica anestésica infiltrativa, incluso en las regiones del paladar y ligamento periodontal, disminuyendo la sensación de dolor en estos tejidos más rígidos (Gibson et al.; Saloum et al., 2000; Primosch \& Brooks; Johnson \& Primosch; Ram \& Peretz, 2003a).
En la literatura, los estudios acerca del método computarizado todavía son pocos y la mayoría se relaciona con la clínica pediátrica, ya que es el público que más miedo tiene del procedimiento. La mayoría de los autores considera la anestesia computarizada tan eficaz como el método convencional, además relatan menor rechazo por parte de los niños a la técnica computarizada, ya que cuando sometidos a ella presentan menos dolor y menor número de quejas (Hochman et al., 1997; Asarch et al., 1999; Allen et al.; Ram e Peretz, 2003b). Sin embargo, Gibson et al., en un estudio similar no observarón diferencias importantes entre las dos técnicas.

Nuestro estudio, a diferencia de los mencionados anteriormente, se llevó a cabo con pacientes adultos, pero los resultados fueron tan satisfactorios como con el público más joven. Sólo el $30 \%$ de los pacientes reportaron alguna molestia en la técnica computarizada y el $70 \%$ informaron que no hubo cualquier dolor, mientras que en la técnica convencional $60 \%$ reportaron molestias y sólo el $40 \%$ informaron que no hubo dolor. Además, algunos pacientes mostraron edema y equimosis postoperatoria en el lado donde se utilizó anestesia convencional, mientras que tras la anestesia computadorizada no se observó ninguna complicación.

En relación al grado de satisfacción del profesional durante la anestesia, aunque los profesionales digan que ambas técnicas son eficaces, lo que está de acuerdo con la literatura, en este estudio afirmaron que aún prefieren la técnica convencional, probablemente porque todavía tengan alguna inhabilidad en el manejo del dispositivo utilizado para la técnica que utiliza inyector anestésico controlado por computador.

En cuanto a cambios hemodinámicos, no hubo diferencias significativas entre las dos técnicas, las pocas alteraciones que fueron observadas se les atribuye a ansiedad o condición personal de los pacientes.

Concluyendo, podemos hacer las siguientes afirmaciones: aunque el grado de satisfacción de los profesionales con la técnica anestésica controlada por computador haya sido significativo, la mayoría declaró preferir el método convencional. No hubo cambios hemodinámicos significativos en cualquiera de los dos métodos de anestesia utilizados en este estudio. La técnica anestésica controlada por computador se mostró sencilla, eficaz y segura. Los pacientes que participaron en este estudio respondieron mejor al método de anestesia controlado por computador, reportando menos dolor. 
BARROS, T. P.; CAMPOLONGO, G.; SEVILHA, F.; DUARTE, D.; BORELLI NETO, L. \& ALVES, N. Estudio comparativo entre la técnica de anestesia local controlada por computador y la técnica de anestesia local convencional. Int. J. Odontostomat., 7(2):175-178, 2013.

BARROS, T. P.; CAMPOLONGO, G.; SEVILHA, F.; DUARTE, D.; BORELLI NETO, L. \& ALVES, N. Comparative study between the conventional local anesthetic technique and the computer-controlled local anesthetic. Int. J. Odontostomat., 7(2):175-178, 2013.

ABSTRACT: Pain control is one of the most important aspects contributing that the patient has a positive behavior in the dental office. However, this still constitutes a challenge for the dentist when treating anxious patients, because the administration of local anesthesia is the cause of many patients to avoid dental treatment. With the purpose of reducing pain sensation for patients and promote greater comfort for patients and professionals was developed a computerized anesthesia injection method. The aim of this investigation was to compare the conventional local anesthetic technique with the computer-controlled local anesthetic. Participated in this study 30 volunteers patients who had indication for bilateral surgical extraction of lower third molars. The patients, randomly selected, were subjected to each of the two mentioned anesthetic techniques (conventional and computer-controlled). Although the degree of professional satisfaction with the computer-controlled local anesthetic technique has been significant, the most of them reported to prefer the conventional method. There was no significant hemodynamic changes in any of the two methods used in this study. The computer-controlled local anesthetic technique was simple, effective and safe. The patients enrolled in this study responded better to the computer-controlled local anesthetic technique, reporting less pain.

KEY WORDS: local anesthesia, anesthesia computerized, control of pain.

\section{REFERENCIAS BIBLIOGRÁFICAS}

Allen, K. D.; Kotil, D.; Larzelere, R. E.; Hutfless, S. \& Beiraghi, S. Comparison of a computerized anesthesia device with a traditional syringe in preschool children. Pediatr. Dent., 24(4):315-20, 2002.

Alves, N. \& Cândido, P. L. Anatomia para o curso de Odontologia geral e específica. $3^{a}$ ed. São Paulo, GenSantos, 2012.

Alves, N. \& Cândido, P. L. Anatomia para o cirurgião-dentista. São Paulo, Gen-Santos, 2008.

Asarch, T.; Allen, K.; Petersen, B. \& Beiraghi, S. Efficacy of a computerized local anesthesia device in pediatric dentistry. Pediatr. Dent., 21(7):421-4, 1999.

Bennett, C. R. M. Anestesia local e controle da dor na prática dentária. $7^{a}$ ed. Rio de Janeiro, Guanabara-Koogan, 1989.

Guedes Pinto, A. C. Odontopediatria. $8^{a}$ ed. São Paulo, GenSantos, 2010.

Gibson, R. S.; Allen, K.; Hutfless, S. \& Beiraghi, S. The Wand vs. traditional injection: a comparison of pain related behaviors. Pediatr. Dent., 22(6):458-62, 2000.

Hochman, M.; Chiarello, D.; Hochman, C. B.; Lopatkin, R. \& Pergola, S. Computerized local anesthetic delivery vs traditional syringe technique. Subjective pain response. N. Y. State Dent. J., 63(7):24-9, 1997.

Johnson, J. \& Primosch, R. E. Influence of site preparation methods on the pain reported during palatal infiltration using the Wand Local Anesthetic System. Am. J. Dent., 16(3):165-9, 2003.

Primosch, R. E. \& Brooks, R. Influence of anesthetic flow rate delivered by The Wand Local Anesthetic System on pain response to palatal injections. Am. J. Dent., 15(1):15-20, 2002.

Ram, D. \& Peretz, B. The assessment of pain sensation during local anesthesia using a computerized local anesthesia (Wand) and a conventional syringe. J. Dent. Child. (Chic.), 70(2):130-3, 2003a.

Ram, D. \& Peretz, B. Assessing the pain reaction of children receiving periodontal ligament anesthesia using a computerized device (Wand). J. Clin. Pediatr. Dent., 27(3):247-50, 2003b.

Saloum, F. S.; Baumgartner, J. C.; Marshall, G. \& Tinkle, J. A clinical comparison of pain perception to The wand and a traditional syringe. Oral Surg. Oral Med. Oral Pathol. Oral Radiol. Endod., 89(6):691-5, 2000.

Vieira, G. F.; Gonçalves, E. A. N. \& Agra, C. M. Anestesia odontológica: segurança e sucesso - Parte 1. Rev . Assoc. Paul. Cir. Dent., 54(1):42-4, 2000.

Walter, L. R. F.; Ferelle, A. \& Issao, M. Condições que interferem na atenção à criança. In: Walter, L. R. F.; Ferelle, A. \& Issao, M. (Eds.). Odontologia para o bebê: odontopediatria do nascimento aos 3 anos. São Paulo, Artes Médicas, 1996.

Wilson, S. Aspectos não-farmacológicos na percepção e controle da dor. In: Pinkham, J. R. (Ed.). Odontopediatria: da infância à adolescência. $2^{\mathrm{a}}$ ed. São Paulo, Artes Médicas, 1996.

Dirección para correspondencia:

Nilton Alves

Departamento de Odontología Integral

Claro Solar, 115, Temuco

CHILE

Email: niltonnalves@yahoo.com.br Recibido: 26-01-2013

Aceptado: 03-04-2013 